Recepción: 12 / 04 / 2018

Aceptación: 08 / 05 / 2018

Publicación: 02 / 07 / 2018

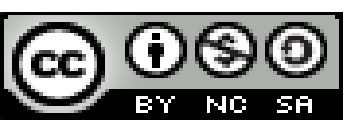

Ciencias de la computación

Artículo de Investigación

\title{
Arquitectura en redes informáticas
}

Architecture in computer networks

Arquitetura em redes de computadores

José R. Caiza-Caizabuano ${ }^{\mathrm{I}}$ jrcaiza@espe.edu.ec
Verónica P. Tintín-Perdomo II vptintin@espe.edu.ec
Hebert L. Atencio-Vizcaino III hhatencio@espe.edu.ec

Correspondencia: jrcaiza@espe.edu.ec

\footnotetext{
I Ingeniero en Sistemas e Informática, Escuela Politécnica del Ejército ESPE, Sangolqui, Pinchincha, Ecuador.

II Ingeniero en Sistemas e Informática, Escuela Politécnica del Ejército ESPE, Sangolqui, Pinchincha, Ecuador.

III Ingeniero en Informática y Sistemas Computacionales, Escuela Politécnica del Ejército ESPE, Sangolqui, Pinchincha, Ecuador.
} 


\section{Resumen}

Teniendo claro que la informática es una ciencia que, con los avances tecnológicos, se vuelve cada vez más compleja; puede inferirse entonces que sus ramas (programación, arquitectura de redes y computadoras, electricidad y electrónica, inteligencia artificial, entre otras) son aún más difusas. La complejidad de las redes informáticas radica en la mezcla de componentes de software y hardware al mismo tiempo. Con el presente artículo se pretende desarrollar el tema de la Arquitectura de Redes Informáticas, básicamente relacionado con un sistema que mediante una infraestructura de interconexión alámbrica o radioeléctrica entre equipos de trasmisión (hardware) y la ejecución de programas y protocolos de comunicación (software), es posible la transferencia de datos, a fines de compartir recursos e información. Este objetivo se estima alcanzar dentro del contexto y desarrollo de una investigación documental a nivel exploratorio, en la que se recurre a material didáctico, accedido de manera física, mediante textos especializados, como también a través de material digital encontrado mediante el motor de búsqueda de Google Académico®. Definitivamente, la idea es que el presente material sirva para aprendizaje básico de cualquier usuario de redes informáticas; asumiendo que estos hoy en día, seguramente tienen una noción de redes informáticas pero que desconocen cómo es que sucede todo ese proceso de envío y recepción de datos a través de impulsos eléctricos, ondas electromagnéticas y similares. Sin embargo, queda a criterio del lector aceptar o rechazar lo aquí expuesto.

Palabras clave: informática; redes; software; interconexión; datos.

\section{Abstract}

Having clear that the computer science is a science that, with the technological advances, it becomes more and more complex; it can be inferred then that its branches (programming, architecture of networks and computers, electricity and electronics, artificial intelligence, among others) are even more diffuse. The complexity of computer networks lies in the mixture of software and hardware components at the same time. This article aims to develop the theme of the Architecture of Computer Networks, basically related to a system that through an infrastructure of wired or radio interconnection between transmission equipment (hardware) and the execution of programs and communication protocols (software), data transfer is possible, in order to share resources and information. This objective is estimated to be achieved within the 
context and development of an exploratory documentary research, in which didactic material is used, accessed in a physical way, through specialized texts, as well as through digital material found through the search engine. Google Scholar ${ }^{\circledR}$. Definitely, the idea is that the present material serves for basic learning of any user of computer networks; assuming that these nowadays, surely they have a notion of computer networks but that they do not know how this process of sending and receiving data happens through electrical impulses, electromagnetic waves and the like. However, it is up to the reader to accept or reject what is stated here.

Keywords: computing; networks; software; interconnection; data.

\section{Resumo}

Tendo claro que a informática é uma ciência que, com os avanços tecnológicos, torna-se cada vez mais complexa; Pode-se inferir, então, que seus ramos (programação, arquitetura de redes e computadores, eletricidade e eletrônica, inteligência artificial, entre outros) são ainda mais difusos. A complexidade das redes de computadores reside na mistura de componentes de software e hardware ao mesmo tempo. Neste artigo tem como objetivo desenvolver o tema da arquitetura de rede, basicamente relacionado a um sistema por meio de infraestrutura de interconexão com fio ou rádio entre equipamentos de transmissão (hardware) e programas de aplicação e protocolos de comunicação (software) A transferência de dados é possível, a fim de compartilhar recursos e informações. Este objectivo deverá ser alcançado dentro do contexto e desenvolvimento de um nível exploratório pesquisa documental, que é usado para materiais de ensino, acessado fisicamente pela textos especializados, bem como através de material digital encontrados pelo motor de busca Google Scholar@. Definitivamente, a idéia é que o presente material serve para aprendizado básico de qualquer usuário de redes de computadores; assumindo que estes hoje em dia, certamente eles têm uma noção de redes de computadores, mas que eles não sabem como este processo de enviar e receber dados acontece através de impulsos elétricos, ondas eletromagnéticas e similares. No entanto, cabe ao leitor aceitar ou rejeitar o que é afirmado aqui.

Palavras chave: Computação; redes; software; interconexão; dados. 


\section{Introducción}

Lo que se conoce hoy en día como redes, tuvo su origen hace ya más de 40 años en los Estados Unidos de América (USA) con la informática centralizada. Ese sistema constaba de un gran ordenador central que se encontraba interconectado con puestos externos (origen de los computadores actuales) desde los que se podía acceder a dicho ordenador centralizado con la finalidad de intercambiar caracteres, que no era más que la compartición de información y servicios.

Los principios de la primera red informática a gran escala data de fin de los años sesenta, cuando el Departamento de Defensa de USA encamina sus esfuerzos en el desarrollo de equipos y software interconectados en una red que funcionara aún si, en caso de guerra, fueran destruidos los equipos tradicionales de comunicación militar, siendo así que nace lo que se conoció como ARPANET (Advanced Research Project Agency NETwork / Agencia de Proyectos de Investigación Avanzada del Departamento de Defensa) apoyándose fundamentalmente para aquel momento, en la red telefónica instalada. A poco tiempo de creación de este sistema (1970) se evidenció un crecimiento acelerado, siendo a escasos dos años que ya contaba con la interconexión de más de cuarenta instituciones estadounidenses que disponían de servicios de correo electrónico y conexión a distancia, y en 1980 se logró la conexión vía satélite con Londres. (Dordoigne, 2015)

Con este preámbulo lo que se quiere dejar ver es que, desde sus inicios a la actualidad, la evolución de las redes informáticas ha sido muy acelerado y divergente, y de la misma manera la arquitectura de esta, (constituida por software y hardware) lógicamente ha tenido que ir desarrollándose a la par de las capacidades que ha demostrado tener el sistema propiamente dicho. En este contexto, teniendo como tema central la arquitectura de redes informáticas, nos avocaremos a describir, en cuanto a su hardware (entendida como la parte, quizás, menos complicada de explicar), los elementos que la componen y las funciones básicas de los mismos; mientras que respecto al software (concebida por el autor de esta obra como el tema más difícil para darse a entender), principalmente se describirá el Modelo ISO/OSI, concebido por Rivera (2016) como "...la base para entender las redes de ordenadores..." (p.5); y se mencionaran otras arquitecturas comúnmente conocidas. 


\section{Materiales y métodos}

De acuerdo con la naturaleza en la obtención de la información, se puede afirmar que el presente trabajo se encuadra dentro de lo que es conocido como investigación documental, y en específico, en cuanto a la profundidad que se pretende alcanzar, se puede decir que la misma es a nivel descriptivo, puesto que la idea que se tiene es, primeramente, desarrollar los conceptos básicos en cuanto a redes informáticas, fundamentales para posteriormente comprender lo relativo a la arquitectura de las redes informáticas. Siendo así, entonces, se puede indicar que para el desarrollo de dicho tema se cuenta con una variedad de material informativo especializado, encontrado tanto en medios físicos como digitales, destacando que, a este último, se pudo acceder a través del motor de búsqueda de Google Académico®. La búsqueda se realizó de manera selectiva, teniendo como criterio las redes informáticas, en primer plano, y la arquitectura de las mismas, en segundo plano, ya que durante la búsqueda y estudio de este tema, se pudo constatar que la arquitectura en redes informáticas deriva de la concepción de los que es una red de computadoras, y fue luego de concebir ese fundamento que se logró seleccionar el contenido deseado, del total del material disponible y consultado, todo ello con la claro objetivo de lograr describir lo que se entiende por redes informáticas, elementos que la componen, características, y arquitectura de las mismas, para que dicha información resulte de mucho interés para aquellos que desean hacer un primer acercamiento a la comprensión de este tema tan complejo.

\section{Resultados}

Para comprender la arquitectura en redes informáticas, se hace necesario, previamente, dar a conocer diversos conceptos asociados a la temática, para así poder facilitar un aprendizaje significativo al respecto, por lo tanto, a continuación, definimos:

\section{Red}

Se refiere, genéricamente, a la interconexión existente de dos o más entidades (representada por objetos o personas) para la circulación e intercambio de elementos materiales o inmateriales, de acuerdo a reglas previamente bien definidas (CCM, 2016). 


\section{Red informática}

"conjunto de equipos conectados por medio de cables, señales, ondas o cualquier otro método de transporte de datos, que comparten información (archivos), recursos (CD-ROM, impresoras) y servicios (acceso a internet, e-mail, chat, juegos), entre otros" (Faure \& García, 2012) (p.28).

\section{Tipos de redes}

Existen varias maneras de clasificar las redes informáticas, sin embargo, fundamentalmente lo que determina cómo sería una estructura (arquitectura) de red es la relación entre ellas, que en definitiva no sería más que la forma en que una computadora utilizaría los recursos de otra a través de la red. Con esto se hace referencia a que las relaciones esenciales entre redes vienen de dos (02) tipos que son: de igual a igual y cliente/servidor.

\section{Relación de red de igual a igual.}

Está definida como una en donde las computadoras pertenecientes a la red se comunican entre sí al mismo nivel, lo que quiere decir que cada computadora es responsable de poner a disposición de los otros ordenadores de la red sus propios recursos, tales como: archivos, directorios, programas de aplicación, impresora, modem, tarjetas de fax; de la misma manera, cada computadora es responsable de configurar y mantener la seguridad de dichos recursos, de acceder a los recursos de red que ésta necesite de otras computadoras de igual a igual, y por último, de conocer dónde se encuentran dichos recursos y qué seguridad se necesita para acceder a los mismos. Cabe destacar que en este tipo de red, es posible tener una computadora dedicada a una función específica de acceder con cierta frecuencia a un recurso de red; ejemplo, podría dedicar a una sola estación de trabajo, la aplicación y losa archivos de datos de un sistema de seguridad de cámaras en circuito cerrado, y no usar esa computadora para realizar las otras tareas típicas de una estación de trabajo, como lo sería el uso del programa de procesamiento de palabras (word, por ejemplo), o el de hojas de cálculo (Excel, por ejemplo), y así concentrar toda la capacidad de la computadora para dicho sistema de seguridad. (Hallberg, 2007)

\section{Relación de red cliente/servidor}

Es aquella en la que se puede diferenciar entre una computadora en la que residen y se administran los recursos de red (llamada: servidor) tales como: archivos, directorios, 
aplicaciones, dispositivos compartidos, entre otros, y aquellas que utilizan dichos recursos, llamadas "cliente o estaciones de trabajo". Cabe destacar que entre las computadoras "cliente" de una red, no se comparten los recursos propios de cada una de ellas como estación de trabaja, ni tampoco con la computadora que funge como servidor, pues estas computadoras son consumidoras de recursos, únicamente.

\section{Características de las redes informáticas}

- Compartición de archivos. Es una tarea que, desde los orígenes, era la razón de ser de las redes informáticas. Esta característica se refiere a la capacidad que proporciona la propia red informática a muchos usuarios de la red al mismo tiempo. Para que ocurra esta tarea, es necesario que en la red se disponga de un directorio compartido o controlador de disco y de una lógica de programación asociada que impida que más de una persona realice cambios conflictivos a un mismo archivo en un mismo momento, de lo contrario, ninguno de los usuarios intervinientes en ese caso, se percataría del conflicto.

- Compartición de impresoras. Es una característica que, tan importante como lo ha sido la compartición de archivos, es primordial en una red informática, puesto que en general, permite abaratar costos. Usualmente, las impresoras de red dependen de un servidor que administras las ya conocidas "colas de impresión", utilidad, eficiente por demás, en las estaciones de trabajo, ya que sus usuarios pueden ordenar las impresiones que necesiten sin tener que esperar a que la impresora culmine las tareas previas asignadas por otros usuarios.

- Servicios de aplicación. Referida a la posibilidad de compartir programas. Esta especialidad permite, cuando se adquiere la licencia adecuada, que un software sea instalado en más de un equipo, y a su vez, la múltiple ejecución de usuarios, entonces, bien podría instalarse un programa en un servidor y poner el contenido del paquete de instalación del mismo a disposición de otros usuarios que requieran del mismo de manera independiente en su propia estación de trabajo, sin que eso signifique que dicho programa no pueda ser ejecutado en el propio servidor, sim embargo, ese tratamiento no sería la única distribución posible, puesto que dependerá de ciertas y determinadas características impuestas por el fabricante de la aplicación, quien por lo general ofrece licencia por usuario, por computadora, o también 
permiten que usuarios de una misma red accedan libremente a la ejecución a partir de una copia del software instalada nativamente, etcétera.

- Correo electrónico. Recurso excelentísimo para las comunicaciones tanto internas como externas de una empresa. Generalmente este tipo de servicio se divide en dos categorías: una que es basada en archivos y la otra llamada cliente/servidor. La diferencia básica entre ellas es que, en el caso del primero de los sistemas mencionados, sólo se encarga de administrar el acceso a una carpeta compartida, contentiva de archivos, ubicada en un servidor tipo, y en el segundo tipo de sistema, se cuenta con un servidor de correo electrónico dedicado, en el que se encuentra el mensaje, y administra el cúmulo de interconexiones tanto de la red a la que pertenece como las de las otras redes externas de correo electrónico a las que esta interconectada, a través de internet, por ejemplo.

- Acceso remoto. Para este servicio hay una amplia gama de sistemas; determinar cuál es el más adecuado, dependerá mayormente de, qué es lo que requieren los usuarios de la red hacer de manera remota, la cantidad total de usuarios y la congruencia de estos regularmente requiriendo de ese servicio, y la cantidad dinero que se pretende gastar en dicho sistema. El acceso remoto no es más que la capacidad que tienen los usuarios de una red informática de acceder a determinados recursos (tales como correo electrónico o archivos) cuando se les imposibilita acceder desde sus propias estaciones de trabajo por cuestiones de distancia, es decir, porque trabajan desde casa, están de viaje, etcétera.

- Redes de área amplia. Son las redes conocidas como WAN (Wide Area Network, por sus siglas en inglés). Esta característica de red está referida a la posibilidad de crear una red de área amplia, mediante la interconexión de una o varias redes informáticas existentes (mayormente LAN, Red de Área Local o Local Area Network, por sus siglas en inglés; o también, MAN / Red de Área Metropolitana o Metropolitan Area Network, por sus siglas en inglés), en razón de que los usuarios de una red LAN necesitan con mucha frecuencia acceder al uso de recursos disponibles en otras redes LAN o MAN.

- Internet e Intranet. Particularidad de la red, vinculada con la idea de que, por una parte, debería existir conectividad entre ésta y el servicio de internet (siendo éste en sí mismo un proveedor de otros servicios de valor agregado, tales como el correo electrónico, la web, el 
acceso a fuentes de información, entre otros); y por la otra, como la interconectividad posible entre los usuarios (clientes) de la red misma, simulando a la internet.

- Seguridad de la red. Relacionada con brindar la seguridad de los recursos de la propia red, de la información que circula a través de ella, y ésta se fundamenta en que tanto los propios usuarios, en sus distintos niveles, junto a la configuración del Sistema Operativo de Red, deben propiciar una combinación de factores que garanticen tanto la estabilidad como la seguridad de la red. (Hallberg, 2007) (pp. 23-28)

\section{Componentes de las redes informáticas}

Como se dijo en un principio, una red informática está compuesta de software y hardware. El Software está referido a lo intangible, a lo relacionado con la operatividad lógica que ocurre por medio del procesamiento de datos existente en los distintos elementos que componen la red, tales como Sistema Operativo de Red, aplicaciones, protocolos de comunicación, entre otros. El hardware está relacionado con los componentes materiales (físicos, tangibles) de una red, tales como computadora (estaciones de trabajo), servidor, tarjeta de interfaz de red, modem, puentes (bridge), enrutador (router), concentradores (hub), switch, cableado, impresora, scaner, nodos, host, entre otros.

\section{Estaciones de trabajo}

Son las computadoras conectadas a la red, con las que se accede a los distintos recursos y servicios proporcionados por la red informática y contenidos en el servidor de archivos de la misma. Cabe destacar que una computadora, como estación de trabajo, no comparte los recursos propios con otras computadoras de la red. (Salazar, 2009)

\section{Servidores}

También son computadoras que poseen ciertas características especiales, tanto de configuración de software como de hardware, para precisamente, como su palabra lo indica, dedicarse a servir o compartir sus singulares recursos con las estaciones de trabajo, siendo dichos recursos: impresoras, unidades de disco, CD-ROM, directorios en disco duro e incluso archivos individuales. (Hallberg, 2007) (pp. 32-33) 


\section{Nodos}

Se trata de un dispositivo físico cualquiera conectado a una red, con capacidad de comunicación hacia otros dispositivos y de compartir tanto los componentes de su hardware como los programas de software, los archivos y datos. Un ejemplo de ello sería lo que comúnmente en un hogar con la interconexión entre una computadora y una impresora, en ese tipo de red simple, ambos dispositivos son nodos, que mediante la conexión existente entre ellos (a través de un cable USB) se pueden comunicar y compartir datos. (Conocimientos Informáticos $\odot, 2014$ )

\section{Host}

Término en inglés que se traduce como "anfitrión", y que en redes informáticas se refiere a cualquier computadora que se encuentre conectada a la red, que posea un número IP (Internet Protocol, por sus siglas en inglés, referido al protocolo de internet, responsable de administrar los paquetes de datos, a través de las redes), lo que le permite enviar y recibir información. (Salazar, 2009)

\section{NIC (Network Interface Card / Tarjeta de Interfaz de Red)}

Es un componente informático que se acopla a la tarjeta madre del CPU de una computadora (estación de trabajo/cliente), contentivo de un transmisor-receptor que prepara los datos que deben transmitirse antes de enviarlos e interpretar los recibidos. Además, contiene un controlador (driver) que se encarga del vínculo entre la tarjeta propiamente dicha y el sistema operativo de red. (Hallberg, 2007) (p. 81)

\section{Hub}

Conocido también concentrador, es un dispositivo que permite la conexión de varios cables de red, definido como punto central de conexión de entrada y salida para nodos de red que están dispuestos de acuerdo a una topología física de estrella. Su función sencillamente es la de unir conexiones y no altera las tramas que le llegan. (López, 2009)

\section{Modem}

Palabra que proviene del acrónimo de "modulador-demodulador", y es un aparato que transforma las señales digitales de la computadora (estación de trabajo) en señal telefónica análoga e 
inversamente, siendo esto lo que le permite a la computadora, a través de una línea telefónica, enviar y recibir (transmitir) información. (López, 2009) (p.19)

\section{Router (Enrutador)}

Tienen la capacidad de conectar tanto redes que son iguales, como redes que son diferentes. Este aparato se convierte en un nodo de red con su propia dirección de red, que recibe los paquetes de otros nodos, los cuales analiza para luego transferirlo a donde corresponde. (Hallberg, 2007) (p.75)

\section{Switch (Conmutador)}

Dispositivo avanzado que combina las funciones de un Hub y de un puente. Tiene la capacidad de reenviar la información solo por el puerto de salida adecuado. (López, 2009) (p.17)

\section{Bridge (Puente)}

Fundamentalmente son "repetidores inteligentes que envían el tráfico de un segmento a otro sólo cuanto este está designado al otro segmento" (Rivera, 2016) (p.68)

\section{Sistema Operativo de Red - SOR (NOS / Network Operating System)}

Es un sistema complejo que permite que varias personas interconectadas (físicamente) trabajen con los mismos recursos compuesto por diferentes capas lógicas (protocolos de comunicación, capa de aplicación, entre otros). Este sistema proporciona un control de acceso a la red (seguridad de conexión, seguridad en el acceso a los recursos, entre otros) coordinando al mismo tiempo los accesos simultáneos (administra a menudo colas de espera para los dispositivos exclusivos). (Hallberg, 2007) (p. 10)

Ahora bien, una vez habiendo adquirido los conceptos antes desarrollados, es importante comprender que a la hora de instalar algún tipo de red, deben tenerse en cuenta los siguientes aspectos: tamaño de la empresa, nivel de seguridad requerido, tipo de actividad, nivel de conocimiento de los administradores de red, volumen de tráfico en la red, necesidades de los usuarios, y el presupuesto destinado al funcionamiento de la red (no solo la compra sino también la actualización y el mantenimiento).

\section{4}




\section{Arquitectura de red}

"Es un sistema funcional compuesto de equipos de transmisión, de programas y protocolos de comunicación y de una de la infraestructura alámbrica o radioeléctrica que permite la transmisión de datos entre los diferentes componentes". (CCM, 2016)

\section{Topología}

Está compuesta por dos partes, la topología física, que es la disposición real de los cables (los medios) y la topología lógica, que define la forma en que los hosts acceden a los medios. De un punto de vista topológico, ésta puede tener forma lineal (bus), de un árbol, de un anillo, de una estrella (jerárquica) o de una malla.

\section{Tipología}

Desde un punto de vista tipológico, la arquitectura es respectivamente calificada como PAN (Personal Area Network) porque su alcance es el más restringido al usuario, es decir, en un área menor a diez metros, aproximadamente; LAN (Personal Area Network) cuando la red se extiende en un perímetro local, menor a $1 \mathrm{~km}$; MAN (Metropolitan Area Network), para comunicación a distancia más extensas, por ejemplo, entre edificios, o un área metropolitana, menor a 100 km; y WAN (Wide Area Network) para distancias mayores que las anteriores, en donde se interconectan varias LAN, e incluso MAN. (CCM, 2016)

Al consultar este aspecto en la literatura desarrollada por Dordoigne (2015) se pudo complementar lo siguiente:

\section{- Red de Área Personal (PAN)}

"Centrada en el usuario, designa una interconexión de equipos informáticos en un espacio de una decena de metros en torno al usuario"

\section{- Red de Área Local (LAN).}

Es de mayor tamaño porque podría expandirse a varios cientos de metros. "Conecta entre sí ordenadores, servidores... Generalmente se utiliza para compartir recursos comunes, como periféricos, datos o aplicaciones." 


\section{- Red de Área Metropolitana (MAN)}

Es una red que "garantiza la comunicación a distancias más extensas y a menudo interconecta varias redes LAN. Puede servir para interconectar, por una conexión privada o pública, diferentes departamentos, distantes algunas decenas de kilómetros".

\section{- Red de Área Extendida WAN}

Están compuestas por redes de tipo LAN, o incluso MAN. Las redes extensas son capaces de transmitir la información a miles de kilómetros por todo el mundo. La WAN más famosa es la red pública Internet, cuyo nombre procede de Inter Networking.

\section{Conclusión}

Compartiendo la idea expuesta al inicio de obra titulada "Fundamentos de Redes Informáticas" de Rivera (2016), basada en el hecho de que: fue a través del desarrollo y evolución de la red informática que se resolvió el problema que existía nativamente de compartir recursos, y además permitió, el surgimiento de un mundo virtual en el que las personas podían trabajar, comunicar, compartir recursos, intercambiar información y hasta mantener un vínculo de amistad, independientemente de la distancia física. (p.7)

El mismo autor asegura que, entre las muchas ventajas que pudiera proporcionar una red informática en un entorno de trabajo, están: la de evasión de trabajo inoficioso, reducción al mínimo del tiempo de dedicación y facilitación del intercambio de información.

Entonces, a la luz de las ideas anteriormente expuestas, es que se puede asegurar se encuentra la importancia de la arquitectura de redes informáticas, puesto que, por medio de la implantación y constante actualización, tanto de la infraestructura como de sus protocolos y sistemas operativos, es que cada vez más se pueden beneficiar, tanto las organizaciones empresariales en sus entornos laborales, como los usuarios particulares que terminan accediendo a la red informática desde un computador personal.

\section{Referencias bibliográficas}

CCM. (03 de 11 de 2016). https://es.ccm.net. (F. C. Benchmark., Editor) Obtenido de https://es.ccm.net/contents/252-el-concepto-de-red 
Conocimientos Informáticos (․ (28 de 01 de 2014). Http://Www.Ordenador.Online. Obtenido de http://www.ordenador.online/Redes/Redes-Locales/Tipos-de-nodos-de-red-.html

Dordoigne, J. (2015). Redes Informáticas. Nociones Fundamentales. Barcelona, España: ENI.

Equipo de Redacción de "Concepto.de". (02 de 2018). www.concepto.de. Recuperado el 01 de 03 de 2018, de http://concepto.de/informatica/

Faure, I., \& García, Y. (2012). Instalaciones de Redes. Vía para fortalecer el aprendizaje de las Redes Informáticas. EduSo, 12(39), 29-35.

Hallberg, B. (2007). Fundamentos de Redes (4 ta. ed.). (C. Cordero, Trad.) Mexico, D.F.: McGraw-Hill / Interamericana Editores, S.A. de C.V. Obtenido de https://issuu.com/torresgtzedgar/docs/fundamentos_de_redes

López, J. (20 de 09 de 2009). https://es.scribd.com. Recuperado el 12 de 01 de 2018, de https://es.scribd.com/presentation/19982677/REDES-INFORMATICAS-Y-CONECTIVIDAD

Rivera, J. (2016). Fundamentos de las Redes Informáticas. Bogotá - Colombia: IT Campus Academy.

Salazar, O. (2009). Glosario de Términos para el Trabajo en Red. Boletín "Entre Líneas"(3-4), 114. Obtenido de http://www.socict.holguin.cu/html/boletines/2008/Dic08/Pdf/01.pdf 